\title{
Efficacy of Dulaglutide in Chinese Patients with Type 2 Diabetes and Different Glycemic Patterns: a Post-hoc Analysis of the Phase 3 AWARD-CHN2 Trial
}

\author{
Qifu Li · Qiqi Zhang · Rui Wang · Tianpei Hong (D)
}

Received: September 7, 2021 / Accepted: November 15, 2021 / Published online: December 6, 2021

(c) The Author(s) 2021

\begin{abstract}
Introduction: We evaluated the effect of dulaglutide on the relative contributions of fasting glucose (FG) and postprandial glucose (PPG) to overall hyperglycemia in patients with type 2 diabetes (T2D), and assessed responses to dulaglutide versus insulin glargine (glargine) in patients with different baseline glycemic patterns.

Methods: This post-hoc analysis of the phase 3 AWARD-CHN2 trial included data from 560 Chinese patients with uncontrolled T2D who received once-weekly dulaglutide $(1.5$ or 0.75 $\mathrm{mg}$ ) or once-daily glargine for 26 weeks. The
\end{abstract}

Supplementary Information The online version contains supplementary material available at https:// doi.org/10.1007/s13300-021-01182-z.

Q. Li

Department of Endocrinology, The First Affiliated Hospital of Chongqing Medical University, Chongqing, China

Q. Zhang · R. Wang $(\bowtie)$

Lilly Suzhou Pharmaceutical Co., Ltd, Shanghai Branch, No. 288 Shimen No.1 Road, Jingan District, Shanghai 200041, China

e-mail: wang_rui1@lilly.com

T. Hong $(\bowtie)$

Department of Endocrinology and Metabolism, Peking University Third Hospital, 49 North Garden Road, Haidian District, Beijing 100191, China e-mail: tpho66@bjmu.edu.cn relative contributions of FG and PPG to overall hyperglycemia across different glycated hemoglobin (HbA1c) categories were calculated using the area under the curve of 7-point self-monitored blood glucose profiles. Patients were also categorized into four subgroups according to median baseline FG (cutoff $8.9 \mathrm{mmol} / \mathrm{L}$ ) and PPG (cutoff $12.5 \mathrm{mmol} / \mathrm{L}$ ): low FG/low PPG, low FG/high PPG, high FG/low PPG and high FG/ high PPG. Changes in glycemic parameters and body weight were calculated for patients in each subgroup.

Results: Among patients receiving dulaglutide, higher HbA1c was associated with higher relative contributions of FG and lower relative contributions of PPG to overall hyperglycemia at baseline and week 26 of dulaglutide treatment. After 26 weeks, dulaglutide $1.5 \mathrm{mg}$ led to statistically greater decreases in HbA1c from baseline versus glargine in most subgroups, including the high FG subgroups, and a numerically greater decrease in HbA1c was observed in the low FG/high PPG subgroup. Across all subgroups, higher proportions of patients achieved $\mathrm{HbA} 1 \mathrm{c} \leq 6.5 \%$ with dulaglutide $1.5 \mathrm{mg}$ than with glargine (all $P<0.05$ ). Dulaglutide $1.5 \mathrm{mg}$ showed better control of body weight than glargine in all subgroups (all $P<0.05)$.

Conclusions: Dulaglutide reduced HbA1c through reductions in both FG and PPG across HbA1c categories in T2D patients with uncontrolled hyperglycemia. Furthermore, treatment 
with dulaglutide provided a greater reduction in HbA1c than glargine, regardless of baseline FG and PPG levels.

Keywords: Dulaglutide; GLP-1 receptor agonist; Basal insulin; Type 2 diabetes; Glycemic pattern

\section{Key Summary Points}

Why carry out this study?

In patients with type 2 diabetes (T2D), the interrelationship of glycated hemoglobin (HbA1c) with fasting glucose (FG) and postprandial glucose (PPG) levels changes with the degree of glycemic control, and there is a need for drugs that impact both fasting and postprandial hyperglycemia.

Limited clinical evidence is currently available to determine the relative contributions of FG and PPG to the overall glucose-lowering effects of glucagon-like peptide- 1 receptor agonists, such as dulaglutide.

This study of Chinese patients with T2D included in the phase 3 AWARD-CHN2 trial evaluated the effect of dulaglutide on the relative contributions of FG and PPG to overall hyperglycemia across $\mathrm{HbA1c}$ categories and assessed treatment responses across patients with different baseline glycemic patterns based on FG and PPG levels.

\section{What was learned from the study?}

Dulaglutide reduced HbA1c through reductions in both FG and PPG across HbA1c categories and provided a greater reduction in HbA1c than insulin glargine, regardless of baseline FG and PPG levels.

The findings of this study support onceweekly dulaglutide as an effective therapeutic option for Chinese patients with T2D inadequately controlled by oral anti-hyperglycemic medications.

\section{INTRODUCTION}

Type 2 diabetes (T2D) is a chronic progressive metabolic disorder characterized by a combination of varied degrees of insulin resistance and secretion deficiency. Since the 1980s, there has been a remarkable increase in the prevalence of diabetes in China-from $0.67 \%$ in 1980 to $11.2 \%$ in 2017 -in association with aging of the population and westernization of diets and lifestyles [1-3]. Diabetes therefore represents a critical health concern in China. It is well established that achieving optimal control of hyperglycemia reduces the risk of long-term diabetes-related complications. Widely accepted treatment guidelines for T2D in both China and other countries are consistent in recommending an individualized treatment approach to achieve target glycated hemoglobin (HbA1c) levels (commonly defined as HbA1c $<7 \%$ ), based upon duration of diabetes, life expectancy, comorbidities, vascular complications and other patient-related factors [3-5].

$\mathrm{HbA1c}$ reflects an average of overall glycemia during both fasting and postprandial states. The interrelationship of HbA1c with fasting glucose (FG) and postprandial glucose (PPG) levels changes during the progression of T2D [6-8]. A study of non-insulin-using patients with T2D showed that the contribution of PPG to overall hyperglycemia decreased across quintiles of HbA1c, increasing from $<7.3 \%$ to $>10.2 \%$, whereas the contribution from FG increased with increasing HbA1c. In patients with HbA1c ranging from $7.3 \%$ to $10.2 \%$, the relative contributions of PPG to overall hyperglycemia ranged approximately from $50 \%$ to $60 \%$ [6].These data indicate that all components of the glucose triad (HbA1c, FG and PPG) should be considered for optimal management of patients with T2D and highlight the need for drugs that impact both FG and PPG.

Generally, patients with T2D inadequately controlled by comprehensive lifestyle modification and oral anti-hyperglycemic medications (OAMs) require initiation of injectable therapy to achieve optimal glycemic control [9]. The phase 3 AWARD-CHN2 trial was conducted to 
compare the efficacy and safety of dulaglutide versus insulin glargine (glargine) in a population of predominantly Chinese patients with T2D and included assessment of HbA1c, PPG and FG. The primary results of the AWARDCHN2 trial showed that dulaglutide $1.5 \mathrm{mg}$ led to greater reductions in $\mathrm{HbA1c}$ with better weight control and a lower incidence of hypoglycemia than glargine [8]. Dulaglutide, a glucagon-like peptide-1 receptor agonist (GLP-1 RA), stimulates insulin secretion and inhibits glucagon release in a glucose-dependent manner in both fasted and postprandial states, and reduces both FG and PPG levels [10]. Other pharmacological effects of GLP-1 RAs include delayed gastric emptying and diminished appetite [11]. Despite these considerations, clinical evidence on the relative contributions of FG and PPG to the overall glucose-lowering effects of GLP-1 RAs is still limited. By contrast, glargine primarily functions by inhibiting hepatic glucose production, consequently lowering FG levels, with only minor effects on PPG [12]. Given these distinct modes of action, it has been hypothesized that patients with different glycemic profiles may respond differently to dulaglutide and glargine treatment. However, there are currently only limited published data to validate this hypothesis in Chinese patients with T2D, and further investigation would be of high value to inform clinical decision-making.

The aims of this post-hoc analysis of the AWARD-CHN2 trial were to evaluate the effect of dulaglutide on the relative contributions of FG and PPG to overall hyperglycemia in Chinese patients with different levels of HbA1c at baseline and at 26 weeks post treatment, and to compare the effect of dulaglutide versus glargine on the relative contribution of FG and PPG to overall hyperglycemia. In addition, the efficacy of dulaglutide versus glargine at 26 weeks was investigated in patients with different baseline FG and PPG categories.

\section{METHODS}

\section{Study Design and Participants}

This was a post-hoc analysis of Chinese patients with T2D randomized in AWARD-CHN2, a phase 3, open-label (blinded to dulaglutide dose), multicenter, non-inferiority study (ClinicalTrials.gov identifier: NCT01648582). The design and primary results of the AWARDCHN2 study have been published [8]. Briefly, the study evaluated the efficacy and safety of once-weekly dulaglutide versus once-daily glargine in adult patients (aged $\geq 18$ years) with a diagnosis of T2D for at least 6 months before screening, uncontrolled hyperglycemia with metformin and/or a sulphonylurea (HbA1c $\geq 7.0$ and $\leq 11.0 \%$ ) and a body mass index (BMI) of $\geq 19.0$ and $\leq 35.0 \mathrm{~kg} / \mathrm{m}^{2}$. Key exclusion criteria were type 1 diabetes, previous receipt of a GLP-1 RA or insulin within 3 months of enrollment, clinically significant gastric emptying abnormalities, history of pancreatitis or serum calcitonin concentration $\geq 20 \mathrm{ng} / \mathrm{L}$. Eligible patients were randomly assigned (1:1:1) to receive once-weekly dulaglutide (1.5 or 0.75 $\mathrm{mg}$ ) or once-daily glargine, for 26 weeks. The AWARD-CHN2 study protocol was approved by the ethics review board of each trial site, including the master ethics review board at Ruijin Hospital Affiliated to Shanghai Jiao Tong University. The study was conducted in line with the ethical principles outlined in the Declaration of Helsinki of 1964 and its later amendments and with local regulations, and all patients provided written informed consent before randomization.

In this post-hoc analysis, the Chinese patients with T2D were categorized according to self-monitored blood glucose (SMBG) levels into four subgroups: low FG/low PPG, low FG/high PPG, high FG/low PPG and high FG/high PPG. The median baseline values of self-monitored 
FG (8.9 mmol/L) and PPG (12.5 mmol/L) among the Chinese patients included in this analysis were used to define the subgroup thresholds (Electronic Supplementary Material [ESM] Fig. 1). It should be noted that these median glucose levels were used as subgroup thresholds because the clinical thresholds of FG $(<7.2$ $\mathrm{mmol} / \mathrm{L})$ and PPG $(<10.0 \mathrm{mmol} / \mathrm{L})$ commonly used to define glycemic control in patients with T2DM [4] led to unbalanced patient numbers in the subgroups.

\section{Measurements}

Efficacy assessments included changes from baseline in HbA1c, FG (central laboratory values), PPG (SMBG values) and the proportion of patients achieving HbA1c targets at week 26. Safety assessments included change in body weight and incidences of overall (plasma glucose $\leq 3.9 \mathrm{mmol} / \mathrm{L}$ ), severe (requiring assistance from another person) and nocturnal hypoglycemia.

\section{Calculation of Glycemic Exposure Over Diurnal Period}

The relative contribution of FG and PPG to overall hyperglycemia was evaluated by calculating fasting hyperglycemia and postprandial hyperglycemia, respectively. Overall hyperglycemia, postprandial hyperglycemia and fasting hyperglycemia were calculated using methods reported previously [13]. In brief, overall hyperglycemia $\left(\mathrm{AUC}_{\text {overall }}\right.$ ) was based on glycemic exposure from the pre-breakfast glucose measurement up to the bedtime glucose measurement and calculated as the area under the curve (AUC) of the 7-point SMBG profiles and above the $5.6 \mathrm{mmol} / \mathrm{L}$ level. Postprandial hyperglycemia $\left(\mathrm{AUC}_{\text {postprandial }}\right)$ was calculated as the sum of the AUC of the 7-point SMBG after each meal, taking each pre-meal level as the baseline. Fasting hyperglycemia $\left(\mathrm{AUC}_{\text {fasting }}\right)$ was then calculated as $\mathrm{AUC}_{\text {overall }}$ minus $\mathrm{AUC}_{\text {postprandial. }}$. The relative contributions of postprandial and fasting hyperglycemia to overall hyperglycemia were calculated across HbA1c categories $(<7 \%, 7$ to $<7.5 \%, 7.5$ to $<$
$8 \%, 8$ to $<8.5 \%, 8.5$ to $<9 \%$ and $\geq 9 \%$ for the baseline measurements and $<6 \%, 6$ to $<6.5 \%$, 6.5 to $<7 \%, 7$ to $<7.5 \%, 7.5$ to $<8 \%$ and $\geq 8 \%$ for measurements at week 26). The relative contributions of postprandial or fasting hyperglycemia were also compared for patients receiving dulaglutide versus glargine across HbA1c quartiles.

\section{Statistical Analysis}

Changes in glycemic outcomes and body weight were calculated in a modified intentionto-treat (mITT) population including all randomized patients with a baseline HbA1c measurement and at least one post-baseline HbA1c measurement and who received at least one dose of study drug. Incidence of hypoglycemia was evaluated in the safety population (all patients receiving at least 1 dose of study drug).

Baseline characteristics were listed in subgroups of patients stratified by median baseline FG/PPG levels. Glycemic and safety outcomes were compared among the baseline FG/PPG subgroups. Continuous variables were presented as mean \pm standard deviation (SD), and discrete variables were presented as the number (percentage), unless otherwise specified. The relative contributions of fasting hyperglycemia and postprandial hyperglycemia within each HbA1c category was compared using a paired Student's $t$ test. The correlation between glycemic outcomes AUC $_{\text {overall }}$ and relative contributions of fasting hyperglycemia and postprandial hyperglycemia) and $\mathrm{HbA} 1 \mathrm{c}$ at baseline and week 26, respectively, were evaluated using a Pearson correlation test. Changes in glycemic outcomes (HbA1c, FG and PPG) at week 26 were analyzed using analysis of covariance (ANCOVA), with treatment, subgroup and treatment by subgroup interaction as fixed effects. Changes in body weight were analyzed using ANCOVA with treatment, subgroup and treatment by subgroup interaction as fixed effects and baseline body weight as a covariate. Results from ANCOVA are summarized as least-squares mean (LSM) with standard error (SE). Missing post-baseline data were imputed using last observation carried forward 
(LOCF). All analyses were performed using SAS version 9.4 software (SAS Institute, Cary, NC, USA).

\section{RESULTS}

\section{Patient Disposition and Baseline Characteristics}

A total of 560 patients were included in this analysis (low FG/low PPG, $n=205$; low FG/high PPG, $n=77$; high FG/low PPG, $n=77$; high FG/ high PPG, $n=201$ ). Overall, around $60 \%$ of patients were male. Mean BMI was $26.03 \mathrm{~kg} / \mathrm{m}^{2}$ and mean age was 54.79 years. The mean baseline $\mathrm{HbA} 1 \mathrm{c}$ level was $8.35 \%$, and the mean duration of diabetes was 8.01 years (Table 1). Demographics and baseline characteristics for the patients further stratified by treatment are presented in ESM Table 1.

\section{Relative Contributions of Fasting and Postprandial Hyperglycemia}

At baseline, there was a significant positive correlation between $\mathrm{AUC}_{\text {overall }}$ (mean $\pm \mathrm{SD}$ : $1374.02 \pm 699.18 \mathrm{mg} \mathrm{h} / \mathrm{dL}$ ) and HbA1c $\left(r^{2}=0.43 ; P<0.001\right)$ among patients receiving dulaglutide $1.5 \mathrm{mg}$. In addition, the relative contribution of fasting hyperglycemia increased (from $40 \%$ to $67 \%$ ) from the lowest to the highest HbA1c category $(<7$ to $\geq 9 \%)\left(r^{2}=0.20\right.$; $P<0.001)$, with a corresponding decrease in the relative contribution of postprandial hyperglycemia (from $60 \%$ to $33 \%$; Fig. 1a).

After 26 weeks of treatment with dulaglutide $1.5 \mathrm{mg}$, the mean $\mathrm{AUC}_{\text {overall }}( \pm \mathrm{SD})$ was $793.94 \pm 452.83 \mathrm{mg} \mathrm{h} / \mathrm{dL}$, which is a reduction from baseline (difference [mean \pm SE] : -580.07 $\pm 56.43 \mathrm{mg} \mathrm{h} / \mathrm{dL} ; P<0.001)$. Similarly, both $\mathrm{AUC}_{\text {fasting }}$ and $\mathrm{AUC}_{\text {postprandial }}$ (mean $\pm \mathrm{SD}$ ) were lower at week 26 compared to baseline

Table 1 Demographics and baseline characteristics of Chinese patients with type 2 diabetes stratified by glycemic pattern

\begin{tabular}{llllll}
\hline Variables & $\begin{array}{l}\text { Low FG/low PPG } \\
(\boldsymbol{n}=\mathbf{2 0 5})\end{array}$ & $\begin{array}{l}\text { Low FG/high } \\
\text { PPG }(\boldsymbol{n}=77)\end{array}$ & $\begin{array}{l}\text { High FG/low } \\
\text { PPG }(\boldsymbol{n}=77)\end{array}$ & $\begin{array}{l}\text { High FG/high } \\
\text { PPG }(\boldsymbol{n}=\mathbf{2 0 1})\end{array}$ & $\begin{array}{l}\text { Overall } \\
(\boldsymbol{n}=\mathbf{5 6 0})\end{array}$ \\
\hline Age, years & $55.62 \pm 9.49$ & $56.57 \pm 10.33$ & $52.64 \pm 9.04$ & $54.07 \pm 9.67$ & $54.79 \pm 9.67$ \\
Male & $128(62.4 \%)$ & $46(59.7 \%)$ & $40(51.9 \%)$ & $120(59.7 \%)$ & $334(59.6 \%)$ \\
Weight, kg & $73.21 \pm 12.32$ & $70.59 \pm 11.70$ & $74.12 \pm 12.82$ & $71.87 \pm 11.87$ & $72.50 \pm 12.17$ \\
$\begin{array}{l}\text { Body mass index, } \\
\text { kg/m }\end{array}$ & $26.13 \pm 3.21$ & $25.42 \pm 3.16$ & $26.69 \pm 3.28$ & $25.91 \pm 3.07$ & $26.03 \pm 3.17$ \\
$\begin{array}{l}\text { Duration of } \\
\quad \text { diabetes, years }\end{array}$ & $7.28 \pm 4.80$ & $8.73 \pm 5.00$ & $7.85 \pm 4.73$ & $8.54 \pm 5.22$ & $8.01 \pm 5.00$ \\
$\begin{array}{l}\text { HbAlc, \% } \\
\text { FG, mmol/L }\end{array}$ & $7.68 \pm 0.75$ & $8.17 \pm 0.90$ & $8.23 \pm 0.88$ & $9.14 \pm 1.01$ & $8.35 \pm 1.08$ \\
PPG ${ }^{\mathrm{a}}, \mathrm{mmol} / \mathrm{L}$ & $10.47 \pm 1.28$ & $13.80 \pm 1.13$ & $11.40 \pm 0.85$ & $15.68 \pm 2.49$ & $12.93 \pm 2.91$ \\
\hline
\end{tabular}

Values are expressed as mean $\pm S D$, with the exception of male sex which is expressed as $n$ (\%)

$F G$ Fasting glucose, $H b A 1 c$ glycated hemoglobin, $P P G$ postprandial glucose, $S D$ standard deviation, $S M B G$ self-monitored blood glucose, $T 2 D$ type 2 diabetes

${ }^{a}$ PPG was based on SMBG 

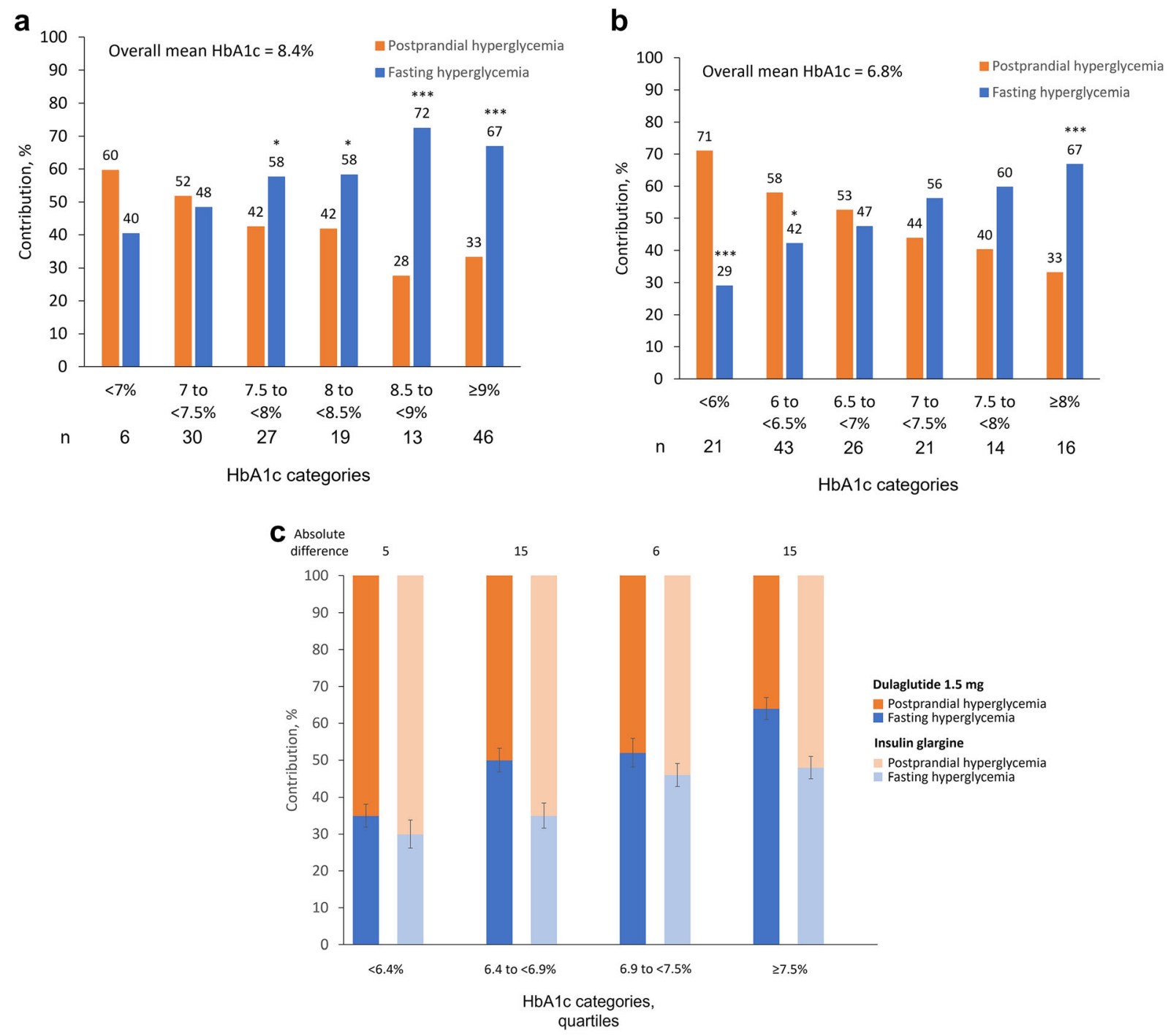

Fig. 1 Relative contributions of fasting glucose and postprandial glucose to overall hyperglycemia stratified by HbAlc categories in Chinese patients receiving dulaglutide $1.5 \mathrm{mg}$ at baseline (a) and week $26(\mathbf{b})$. c Comparison of patients receiving dulaglutide $1.5 \mathrm{mg}$ and those receiving

AUC $_{\text {fasting }}$ at week $26: 417.83 \pm 354.05 \mathrm{mg}$ $\mathrm{h} / \mathrm{dL} ; \quad$ difference [mean $\pm \mathrm{SE}]:-440.60 \pm$ $47.00 \mathrm{mg} \mathrm{h} / \mathrm{dL}, P<0.001 ; \mathrm{AUC}_{\text {postprandial }}$ at week 26: $376.11 \pm 204.77 \mathrm{mg} \mathrm{h} / \mathrm{dL}$, difference $[$ mean \pm SE] $:-139.48 \pm 23.00 \mathrm{mg} \mathrm{h} / \mathrm{dL}$, $P<0.001)$. In line with the baseline observations, $\mathrm{AUC}_{\text {overall }}$ showed a significant positive correlation with HbA1c $\left(r^{2}=0.42 ; P<0.001\right)$ after treatment with dulaglutide $1.5 \mathrm{mg}$. Also consistent with the trend observed at baseline, glargine, at week 26. Asterisks denote significant difference between fasting and postprandial hyperglycemia within each HbAlc category at ${ }^{*} P<0.05,{ }^{* *} P<0.01$ and ${ }^{* * *} P<0.001$. HbAlc Glycated hemoglobin

after dulaglutide treatment, the relative contribution of fasting hyperglycemia to overall hyperglycemia increased (from 29 to 67\%) from the lowest to highest HbA1c categories $(<6$ to $\geq$ $\left.8 \% ; r^{2}=0.27, P<0.001\right)$ with a reciprocal decrease in the contribution of postprandial hyperglycemia (from 71 to 33\%) (Fig. 1b).

Patients receiving dulaglutide $1.5 \mathrm{mg}$ and glargine achieved significant reductions in mean HbA1c from baseline to week 26. After 26 
weeks of treatment with glargine, the mean $\mathrm{AUC}_{\text {overall }}( \pm \mathrm{SD})$ was $887.07 \pm 444.53 \mathrm{mg}$ $\mathrm{h} / \mathrm{dL}$. Consistent with the known basal-preferential effect of glargine, patients receiving glargine had a lower relative contribution to overall hyperglycemia from fasting hyperglycemia but a higher relative contribution from postprandial hyperglycemia across all quartiles of $\mathrm{HbA1c}$ following 26 weeks of treatment (Fig. 1c). Similar results were observed for patients receiving dulaglutide $0.75 \mathrm{mg}$ (ESM Fig. 2; ESM Table 2).

\section{Changes in HbA1c from Baseline}

At week 26, significant reductions in LSM $\mathrm{HbA1c}$ from baseline were observed for patients receiving dulaglutide $1.5 \mathrm{mg}$ and glargine across all subgroups (all $P<0.05$ ) (Fig. 2 a). Overall, the greatest reductions were observed in the high FG/high PPG subgroup (dulaglutide $1.5 \mathrm{mg}$ :$2.22 \%$; glargine: $-1.55 \%)$. A significantly greater reduction in LSM HbA1c from baseline was observed for patients receiving dulaglutide $1.5 \mathrm{mg}$ versus glargine in the low FG/low PPG (LSM difference: $-0.70 \%, \quad 95 \%$ confidence interval $[95 \% \mathrm{CI}]-1.04,-0.37 ; P<0.001)$, high FG/low PPG (LSM difference: $-0.85 \%$, 95\% CI $-1.42,-0.28 ; P=0.003)$ and high FG/ high PPG (LSM difference: - 0.67\%, 95\% CI $1.00,-0.34 ; P<0.001$ ) subgroups (Fig. 2a). Among patients receiving dulaglutide $0.75 \mathrm{mg}$, a significant reduction in LSM HbA1c from baseline was also observed across all subgroups. Patients receiving dulaglutide $0.75 \mathrm{mg}$ had a numerically higher reduction in HbA1c from baseline than patients receiving glargine, although the differences did not reach statistical significance (ESM Fig. 3a).

\section{Proportion of Patients Achieving HbA1c Targets}

At week 26, HbA1c $<7 \%$ was achieved by a greater proportion of patients receiving dulaglutide $1.5 \mathrm{mg}$ versus glargine across all subgroups, and the difference reached statistical significance in the low FG/low PPG, low FG/ high PPG and high FG/high PPG subgroups (Fig. 2b). In addition, a significantly higher proportion of patients receiving dulaglutide 1.5 $\mathrm{mg}$ achieved $\mathrm{HbA} 1 \mathrm{c} \leq 6.5 \%$ versus glargine across all subgroups (Fig. 2c). Similar trends were observed for patients receiving dulaglutide $0.75 \mathrm{mg}$, with significantly higher proportions of patients achieving HbA1c $<7.0 \%$ and $\leq 6.5 \%$ in the low FG/low PPG subgroup (ESM Fig. 3b, c).

\section{Changes in FG and PPG from Baseline}

Statistically significant LSM reductions in FG from baseline to week 26 were observed for patients receiving dulaglutide $1.5 \mathrm{mg}$ and glargine across all subgroups (Fig. 2d). The greatest reductions in FG were achieved in patients belonging to the high FG/high PPG subgroup and the smallest reductions in those belonging to the low FG/low PPG subgroup. Differences in reduction of FG from baseline to week 26 between the dulaglutide $1.5 \mathrm{mg}$ and glargine treatment groups did not reach statistical significance across all subgroups.

Statistically significant LSM reductions in PPG were observed at week 26 for patients receiving dulaglutide $1.5 \mathrm{mg}$ and glargine across all subgroups, with the exception of patients receiving glargine in the low FG/low PPG subgroup (Fig. 2e). The greatest and smallest reductions in PPG were achieved in patients belonging to the high FG/high PPG subgroup and low FG/low PPG subgroup, respectively. Overall, patients treated with dulaglutide 1.5 $\mathrm{mg}$ tended to achieve a greater reduction in PPG than those receiving glargine across all subgroups, and the difference reached statistical significance in the high FG/high PPG subgroup (LSM difference: - $1.43 \mathrm{mmol} / \mathrm{L}, 95 \%$ CI $2.24,-0.62 ; P<0.001)$.

A consistent trend was observed among patients receiving dulaglutide $0.75 \mathrm{mg}$, with a significantly greater reduction in PPG at week 26 versus patients receiving glargine in the low FG/low PPG subgroup ( -1.35 vs. $-0.45 \mathrm{mmol} /$ L; LSM difference: $-0.90 \mathrm{mmol} / \mathrm{L}, 95 \% \mathrm{CI}-$ 1.71, $-0.10 ; P=0.028$; ESM Fig. 3d, e). 

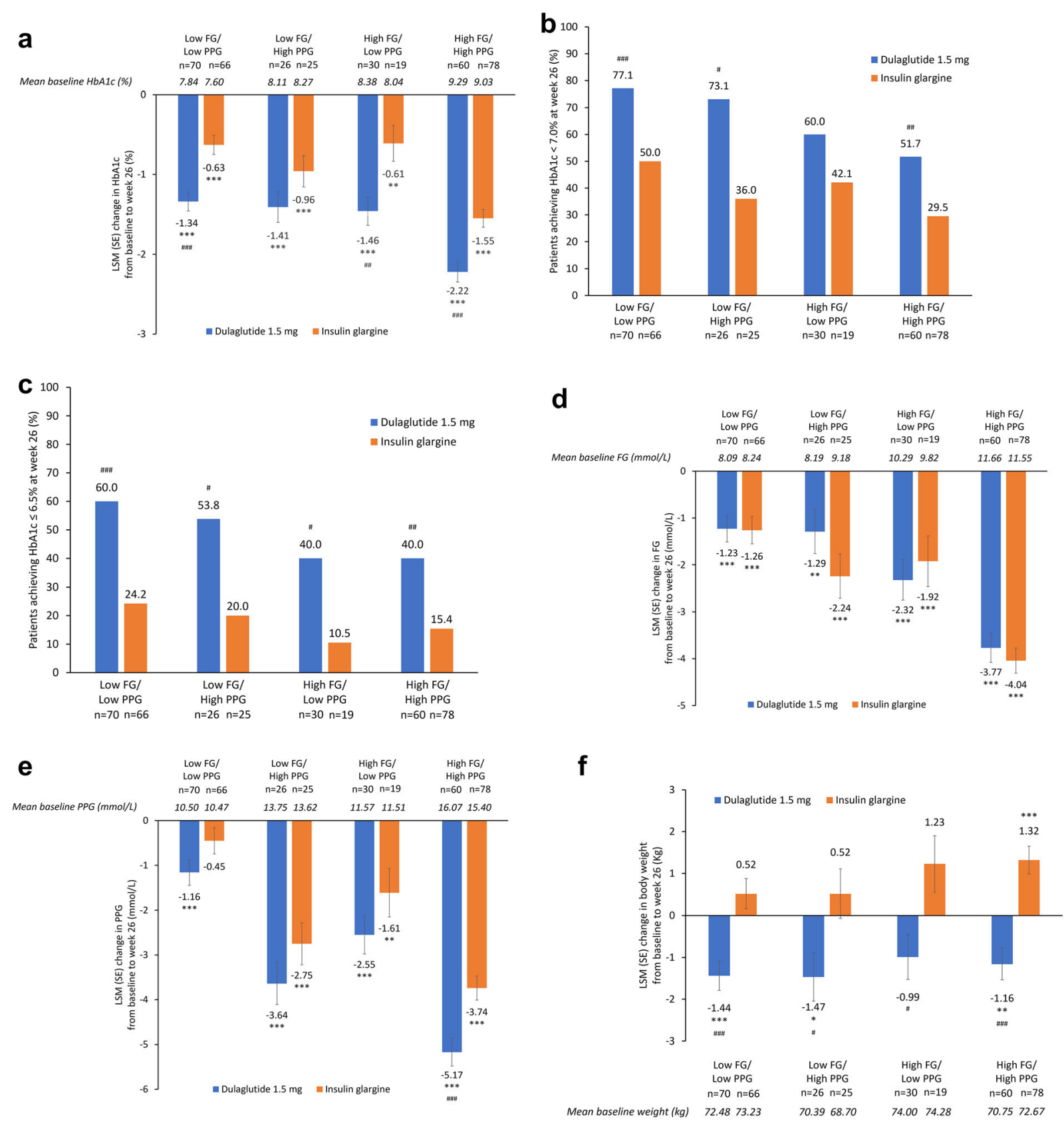

Fig. 2 Glycemic control and body weight change at week 26 stratified by glycemic pattern in Chinese patients with T2D treated with dulaglutide $1.5 \mathrm{mg}$ or glargine. a Change in $\mathrm{HbAlC}$ from baseline, $\mathbf{b}$ proportion of patients with $\mathrm{HbAlc}<7.0 \%$, c proportion of patients with $\mathrm{HbAlc} \leq$ $6.5 \%, \mathrm{~d}$ change in FG from baseline, e change in PPG glucose from baseline, $\mathbf{f}$ change in body weight from baseline. Asterisks denote denote significant difference

from baseline at ${ }^{*} P<0.05,{ }^{* *} P<0.01$ and ${ }^{* * *} P<0.001$. Hash signs indicate significant difference between dulaglutide $1.5 \mathrm{mg}$ and glargine within each glycemic pattern category at ${ }^{\#} P<0.05,{ }^{\# \#} P<0.01$ and ${ }^{\# \#} P<0.001 . F G$ fasting glucose, $L S M$ least-squares mean, $P P G$ postprandial glucose, $S E$ standard error, $T 2 D$ type 2 diabetes 


\section{Change in Body Weight from Baseline}

Dulaglutide $1.5 \mathrm{mg}$ led to significantly better control of body weight than glargine in all subgroups: low FG/low PPG (LSM difference: $-1.97 \quad \mathrm{~kg}, \quad 95 \% \quad \mathrm{CI}-2.96,-0.98$; $P<0.001$ ), low FG/high PPG (LSM difference: $-1.98 \quad \mathrm{~kg}, \quad 95 \% \quad \mathrm{CI}-3.60,-0.37$; $P=0.016$ ), high FG/low PPG (LSM difference: $-2.22 \quad \mathrm{~kg}, \quad 95 \% \quad \mathrm{CI}-3.91,-0.53$; $P=0.010$ ) and high FG/high PPG (LSM difference: $-2.49 \quad \mathrm{~kg}, \quad 95 \% \quad \mathrm{CI}-3.48,-1.50$; $P<0.001$ ) subgroups (Fig. 2f). A similar trend was observed in patients treated with dulaglutide $0.75 \mathrm{mg}$, with significant treatment differences observed in the low FG/low PPG, high FG/ low PPG and high FG/high PPG subgroups (ESM Fig. 3f).

\section{Incidence of Hypoglycemia}

At week 26, the incidence of overall and nocturnal hypoglycemia was lower among patients receiving dulaglutide $1.5 \mathrm{mg}$ versus glargine across all glycemic pattern subgroups, apart from the low FG/high PPG subgroup in which the incidence of overall and nocturnal hypoglycemia was comparable for the two treatments (29.6 vs. $28.0 \%$ and 7.4 vs. $8.0 \%$, respectively) (Table 2). No patient experienced severe hypoglycemia during the trial. Similar results were observed for patients receiving dulaglutide $0.75 \mathrm{mg}$ (ESM Table 3 ). The mean glargine dose at week 26 was higher in the high FG groups (high FG/low PPG: 27.42 U; high FG/ high PPG: $24.80 \mathrm{U}$ ) than in the low FG groups (low FG/low PPG: $16.21 \mathrm{U}$; low FG/high PPG: $18.00 \mathrm{U})$.

\section{DISCUSSION}

This post-hoc analysis of the AWARD-CHN2 trial is the first study to investigate the effect of a GLP-1 RA on glycemic control deep-dived from glycemic patterns in a population of Chinese patients with T2D. The results of this analysis showed that the relative contribution of FG to overall hyperglycemia increased, and the relative contribution of PPG decreased, with increasing baseline HbA1c levels, and that this trend was maintained after 26 weeks of treatment with dulaglutide. This finding suggests that dulaglutide lowers both fasting and postprandial hyperglycemia to reduce HbA1c levels. Furthermore, dulaglutide provided a greater reduction in $\mathrm{HbA1c}$ than glargine, regardless of baseline FG and PPG levels. Taken together, these data indicate that dulaglutide is an effective glucose-lowering therapeutic option for Chinese patients with T2D.

As reported in a previous study conducted in French patients with T2D inadequately controlled by OAMs, the relative contribution of postprandial hyperglycemia to overall glycemic load varies according to the degree of glycemic control [6]. The relative contribution of PPG is predominant in patients with fairly well controlled glycemia, whereas the relative contribution of FG increases gradually with worsening of glycemic control [6]. For example, in patients with HbA1c $<7.3 \%$, PPG contributed to $69.7 \%$ of overall hyperglycemia, and in patients with HbA1c of $7.3-10.2 \%$, the relative contributions of FG and PPG to overall hyperglycemia were balanced (approx. 50\% each); in contrast, in poorly controlled patients with $\mathrm{HbA} 1 \mathrm{c}>$ $10.2 \%$, the relative contribution of PPG decreased to $30.5 \%$ [6]. This post-hoc analysis showed that dulaglutide $1.5 \mathrm{mg}$ reduced $\mathrm{HbA} 1 \mathrm{c}$, and the relative contribution of PPG to overall hyperglycemia after 26 weeks of treatment ranged from 71 to $33 \%$ across HbA1c categories $(<6.0$ to $\geq 8.0 \%)$. This finding indicates that dulaglutide has a proportional impact on both FG and PPG across different HbA1c levels. In addition, patients receiving dulaglutide showed a higher FG contribution and lower PPG contribution than those receiving glargine, across all HbA1c quartiles. Our findings are consistent with a pooled analysis of five phase 3 trials conducted in predominantly Caucasian patients with T2D inadequately controlled by one to two OAMs [13]. Furthermore, in line with previous findings in predominantly Caucasian patients with T2D [7], the basal preferential effect of glargine in Chinese patients with T2D was evident from the narrow range of relative contributions of FG to overall 


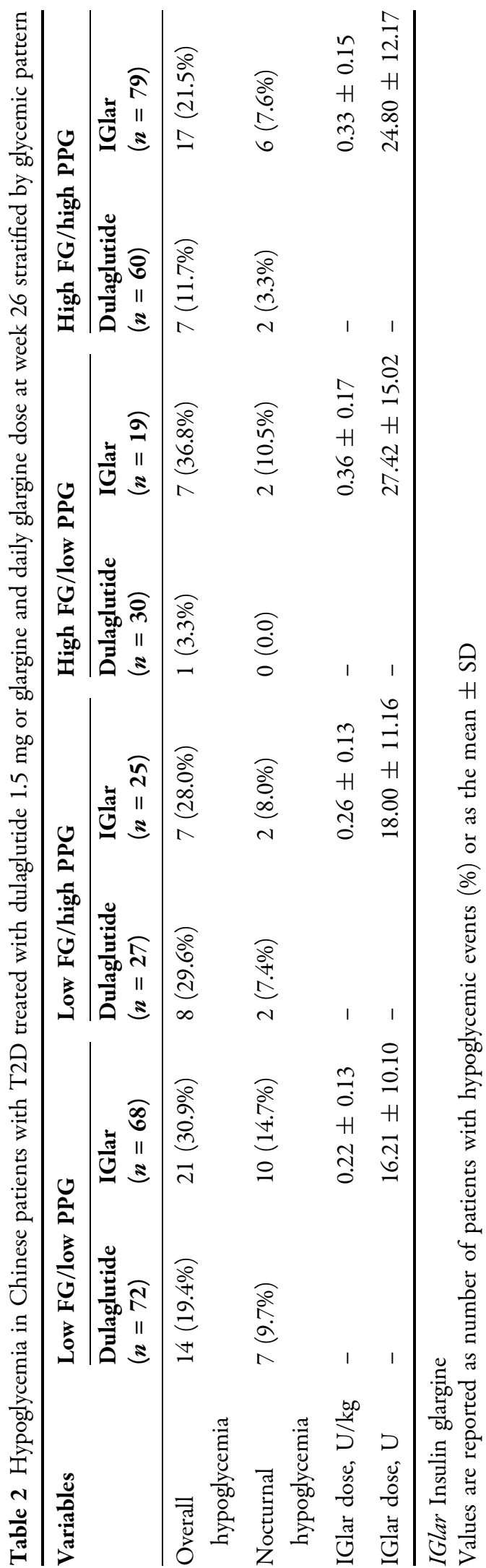

hyperglycemia (30-48\%) observed across HbA1c categories $(<6.4$ to $\geq 7.5 \%)$.

In the present analysis, dulaglutide led to greater HbA1c reductions than glargine across the different baseline FG/PPG subgroups. As expected, the greatest reduction in mean HbA1c level was observed in the high FG/high PPG subgroup and a higher baseline HbA1c level was associated with greater $\mathrm{HbA1c}$ reductions. However, compared with glargine, dulaglutide was also associated with an increased HbA1c reduction in the high FG/low PPG subgroup. These data further support the premise that glycemic control can be improved by utilizing therapies targeting both PPG and FG. Previous observations indicate that particular attention should be paid to the management of PPG in patients with near-normal blood glucose concentrations in order to reach individualized HbA1c targets [6]. Thus, it seems reasonable to implement therapies with a PPG-lowering effect, especially when prandial hyperglycemic load is preeminent.

Compared with a post-hoc analysis of the AWARD-2 trial conducted in predominantly Caucasian patients with T2D [14], this post-hoc analysis showed that dulaglutide led to greater reductions in HbA1c in Chinese patients across all baseline glycemic pattern subgroups. In addition, a meta-analysis of 15 clinical trials showed a greater reduction in HbA1c with GLP1 RAs versus placebo among predominantly Asian patients compared to non-Asian patients (weighted mean difference: $-1.16 \%$ [95\% $\mathrm{CI}-1.48,-0.85] \quad$ vs. $-0.83 \% \quad[95 \% \quad \mathrm{CI}-$ $0.97,-0.70]$; between group difference: $-0.32 \%, \quad 95 \% \quad C I-0.64,-0.01$; $P=0.04$ ) [15]. However, the underlying explanation for differences in dulaglutide efficacy between Chinese patients with T2D and patients from other regions is not fully understood. Further studies are needed to investigate if dulaglutide is a preferred option for Chinese patients with T2D.

Consistent with prior findings in predominantly Caucasian patients with T2D [14], this post-hoc analysis in Chinese patients with T2D indicated that dulaglutide led to weight loss, versus weight gain with glargine, regardless of baseline FG and PPG levels. Compared with 
patients treated with glargine, a lower incidence of overall and nocturnal hypoglycemia was observed in patients treated with dulaglutide across all subgroups except those with low FG/ high PPG at baseline, in whom the incidence was comparable in patients receiving both treatments. In addition to the glucose-lowering and weight loss effects and low risk of hypoglycemia associated with dulaglutide that were supported by the present analysis, the primary and secondary cardiovascular benefits of dulaglutide [16] as well as frequency of administration and adherence are clinically relevant factors that should be considered when choosing anti-hyperglycemic therapies following a patient-centric approach $[17,18]$.

Given that glargine was titrated according to FG in the AWARD-CHN2 trial, higher doses were observed at 26 weeks in the high FG subgroups. As shown in the primary report of the AWARD-CHN2 trial, the mean daily dose of glargine at 26 weeks was $0.29 \mathrm{U} / \mathrm{kg}$ [8]. Accordingly, the mean daily doses of glargine (range $0.22-0.36 \mathrm{U} / \mathrm{kg}$ ) observed in this posthoc analysis were comparable to those reported in the phase III ABET trial $(0.22 \mathrm{U} / \mathrm{kg})$, in which Chinese patients with T2D inadequately controlled by $\geq 2$ OAMs received glargine for 24 weeks [19], but were lower than reports from two similar international phase 3 trials conducted in Caucasian and multiracial patients with T2D $(0.48-0.61 \mathrm{U} / \mathrm{kg})[20,21]$.

This study had several limitations, including the post hoc nature of the analysis. The relatively short duration of 26 weeks may not represent the long-term effects of dulaglutide, although consistent results were reported in the post hoc analysis of the AWARD-2 study with a 52-week duration [14]. A potential bias in the evaluation of fasting and postprandial hyperglycemia may have been caused by the single "point-in-time" measurement of SMBG and thus an inability to provide an accurate estimate of glucose exposure. In this regard, future studies using continuous glucose monitoring are warranted. Additionally, the use of median FG and PPG levels as cutoffs resulted in a less strict definition of glycemic patterns and may limit the generalizability of the results of this study. However, it was not possible to use the clinically accepted cutoffs for FG and PPG as it would have resulted in very small patient numbers in the low FG/high PPG and high FG/ low PPG subgroups. Finally, the generalizability of the findings of this analysis may be less robust in patients with $\mathrm{HbA} 1 \mathrm{c}>11 \%$ due to the characteristics of the pre-defined study population.

\section{CONCLUSIONS}

In summary, the present analysis further strengthens current evidence that dulaglutide reduces HbA1c by lowering both fasting and postprandial hyperglycemia across a wide range of HbA1c levels. Interestingly, a trend for better glycemia and weight control and lower risk of hypoglycemia was observed in patients treated with dulaglutide versus those receiving glargine regardless of baseline FG and PPG levels. Taken together with the potential cardioprotective effect of dulaglutide, these findings support the clinical use of once-weekly dulaglutide as an optimal option for Chinese patients with T2D inadequately controlled by 1-2 OAMs.

\section{ACKNOWLEDGEMENTS}

Funding. This study was funded by Eli Lilly and Company. The Rapid Service fee was also paid for by Eli Lilly and Company.

Medical Writing, Editorial, and other Assistance. The authors thank Huan Li and Chengwei Li, from Eli Lilly and Company, for data analysis for Fig. 1 and ESM Fig. 2, and Ying Lou and Haiya $\mathrm{Wu}$, from Eli Lilly and Company, for their valuable review of this article. The authors also thank Nan Yao, from Eli Lilly and Company, for project management, medical writing and editorial assistance. The authors thank Jake Burrell from Rude Health Consulting for medical writing support and editorial assistance.

Authorship. All named authors meet the International Committee of Medical Journal 
Editors (ICMJE) criteria for authorship for this article, take responsibility for the integrity of the work as a whole and have given their approval for this version to be published.

Author Contributions. Rui Wang was responsible for the study concept and design and interpretation of data. Tianpei Hong was responsible for interpretation of data, original drafting of the article and critical revision for important intellectual content. Qifu Li was involved in the acquisition and interpretation of data. Qiqi Zhang was responsible for the statistical considerations in the analysis. All authors reviewed and gave final approval of the version to be published and agree to take responsibility for the integrity of the work as a whole in ensuring that questions related to the accuracy or integrity of any part of the work are appropriately investigated and resolved.

Disclosures. Rui Wang and Qiqi Zhang are employees of Eli Lilly and Company. Qifu Li and Tianpei Hong declare they have nothing to disclose.

Compliance with Ethics Guidelines. The AWARD-CHN2 study protocol was approved by the ethics review board of each trial site, including the master ethics review board at Ruijin Hospital Affiliated to Shanghai Jiao Tong University. The study was conducted in line with the ethical principles outlined in the Declaration of Helsinki of 1964 and its later amendments and with local regulations, and all patients provided written informed consent before randomization.

Data Availability. The datasets generated during and/or analyzed during the current study are available from the corresponding authors on reasonable request.

Open Access. This article is licensed under a Creative Commons Attribution-NonCommercial 4.0 International License, which permits any non-commercial use, sharing, adaptation, distribution and reproduction in any medium or format, as long as you give appropriate credit to the original author(s) and the source, provide a link to the Creative Commons licence, and indicate if changes were made. The images or other third party material in this article are included in the article's Creative Commons licence, unless indicated otherwise in a credit line to the material. If material is not included in the article's Creative Commons licence and your intended use is not permitted by statutory regulation or exceeds the permitted use, you will need to obtain permission directly from the copyright holder. To view a copy of this licence, visit http://creativecommons.org/licenses/by$\mathrm{nc} / 4.0 /$.

\section{REFERENCES}

1. Li Y, Teng D, Shi X, et al. Prevalence of diabetes recorded in mainland China using 2018 diagnostic criteria from the American Diabetes Association: national cross sectional study. BMJ. 2020;369: m997.

2. Jia W, Weng J, Zhu D, et al. Standards of medical care for type 2 diabetes in China 2019. Diabetes Metab Res Rev. 2019;35(6):e3158.

3. Chinese Diabetes Society. Guideline for the prevention and treatment of type 2 diabetes mellitus in China (2020 edition) [Chinese]. Chin J Diabetes Mellitus. 2021;13(4):315-409.

4. American Diabetes Association. 6. Glycemic targets: standards of medical care in diabetes-2021. Diabetes Care. 2021;44(Suppl 1):S73-84.

5. Action to Control Cardiovascular Risk in Diabetes Study Group, Gerstein HC, Miller ME, et al. Effects of intensive glucose lowering in type 2 diabetes. N Engl J Med. 2008;358(24):2545-59.

6. Monnier L, Lapinski H, Colette C. Contributions of fasting and postprandial plasma glucose increments to the overall diurnal hyperglycemia of type 2 diabetic patients: variations with increasing levels of HbA(1c). Diabetes Care. 2003;26(3):881-5.

7. Riddle M, Umpierrez G, DiGenio A, Zhou R, Rosenstock J. Contributions of basal and postprandial hyperglycemia over a wide range of A1C levels before and after treatment intensification in type 2 diabetes. Diabetes Care. 2011;34(12):2508-14.

8. Wang W, Nevárez L, Filippova E, et al. Efficacy and safety of once-weekly dulaglutide versus insulin glargine in mainly Asian patients with type 2 
diabetes mellitus on metformin and/or a sulphonylurea: a 52-week open-label, randomized phase III trial. Diabetes Obes Metab. 2019;21(2):234-43.

9. American Diabetes Association. 9. Pharmacologic approaches to glycemic treatment: standards of medical care in diabetes-2021. Diabetes Care. 2021;44(Suppl 1):S111-24.

10. Aroda VR. A review of GLP-1 receptor agonists: evolution and advancement, through the lens of randomised controlled trials. Diabetes Obes Metab. 2018;20(Suppl 1):22-33.

11. Drucker DJ. Mechanisms of action and therapeutic application of glucagon-like peptide-1. Cell Metab. 2018;27(4):740-56.

12. Cahn A, Miccoli R, Dardano A, Del Prato S. New forms of insulin and insulin therapies for the treatment of type 2 diabetes. Lancet Diabetes Endocrinol. 2015;3(8):638-52.

13. Umpierrez G, Pantalone KM, Atisso CM, Landó LF, Patel H. Relative contribution of basal and postprandial hyperglycaemia stratified by $\mathrm{HbA1c}$ categories before and after treatment intensification with dulaglutide. Diabetes Obes Metab. 2019;21(6): 1365-72.

14. Giorgino F, Yu M, Haupt A, Milicevic Z, GarcíaPérez LE. Effect of once-weekly dulaglutide versus insulin glargine in people with type 2 diabetes and different baseline glycaemic patterns: a post hoc analysis of the AWARD-2 clinical trial. Diabetes Obes Metab. 2019;21(11):2570-5.

15. Kim YG, Hahn S, Oh TJ, Park KS, Cho YM. Differences in the HbA1c-lowering efficacy of glucagonlike peptide- 1 analogues between Asians and nonAsians: a systematic review and meta-analysis. Diabetes Obes Metab. 2014;16(10):900-9.
16. Gerstein HC, Colhoun HM, Dagenais GR, et al. Dulaglutide and cardiovascular outcomes in type 2 diabetes (REWIND): a double-blind, randomised placebo-controlled trial. Lancet. 2019;394(10193): 121-30.

17. Garber AJ, Handelsman Y, Grunberger G, et al. Consensus statement by the American Association of Clinical Endocrinologists and American College of Endocrinology on the comprehensive type 2 diabetes management algorithm-2020 executive summary. Endocr Pract. 2020;26(1):107-39.

18. Davies MJ, D'Alessio DA, Fradkin J, et al. Management of hyperglycemia in type 2 diabetes, 2018. A consensus report by the American Diabetes Association (ADA) and the European Association for the Study of Diabetes (EASD). Diabetes Care. 2018;41(12):2669-701.

19. Feng W, Chen W, Jiang S, Du L, Zhu D. Efficacy and safety of LY2963016 insulin glargine versus insulin glargine (Lantus) in Chinese adults with type 2 diabetes: a phase III, randomized, open-label, controlled trial. Diabetes Obes Metab. 2021;23(8): 1786-94.

20. Pollom RK, Ilag LL, Lacaya LB, Morwick TM, Ortiz CR. Lilly insulin glargine versus Lantus( $(\mathbb{R})$ in insulin-naïve and insulin-treated adults with type 2 diabetes: a randomized, controlled trial (ELEMENT 5). Diabetes Ther. 2019;10(1):189-203.

21. Rosenstock J, Hollander P, Bhargava A, et al. Similar efficacy and safety of LY2963016 insulin glargine and insulin glargine (Lantus ${ }^{\circledR}$ ) in patients with type 2 diabetes who were insulin-naïve or previously treated with insulin glargine: a randomized, double-blind controlled trial (the ELEMENT 2 study). Diabetes Obes Metab. 2015;17(8):734-41. 